\title{
Seven-Year Surveillance of emm Types of Pediatric Group A Streptococcal Pharyngitis Isolates in Western Greece
}

\author{
George A. Syrogiannopoulos ${ }^{1 *}$, loanna N. Grivea ${ }^{1}$, Adnan Al-Lahham ${ }^{2,3}$, Maria Panagiotou ${ }^{1,4}$, \\ Alexandra G. Tsantouli ${ }^{1}$, Aspasia N. Michoula Ralf René Reinert ${ }^{1,2 \alpha}$, Mark van der Linden ${ }^{2}$ \\ 1 University of Thessaly, School of Health Sciences, Faculty of Medicine, General University Hospital of Larissa, Biopolis, Larissa, Greece, 2 Institute for Medical Microbiology \\ and National Reference Center for Streptococci, University Hospital, Aachen, Germany, 3 School of Applied Medical Sciences, German Jordanian University, Amman, \\ Jordan, 4 University of Patras, School of Health Sciences, Faculty of Medicine, General University Hospital of Patras, Rion, Patras, Greece
}

\begin{abstract}
Background: An experimental 26-valent M protein Group A streptococcal (GAS) vaccine has entered clinical studies. Pharyngeal GAS emm type surveillances in different areas and time-periods enhance the understanding of the epidemiology of GAS pharyngitis. Moreover, these surveillances, combined with the data on GAS invasive disease, can play a significant role in the formulation of multivalent type-specific vaccines.

Methods: During a 7-year period (1999-2005), 2408 GAS isolates were recovered from consecutive children with pharyngitis in Western Greece. The overall macrolide resistance rate was $22.8 \%$. Along the study period we noted a tendency towards significantly decreased rates of resistance, with the lowest rates occurring in 2002 (15.3\%), 2003 (15\%) and 2004 (16.7\%). A random sample of isolates from each year, $338(61.7 \%)$ of the 548 macrolide-resistant and $205(11 \%)$ of the macrolidesusceptible, underwent molecular analysis, including emm typing.

Results: The 543 typed isolates had 28 different emm types. A statistically significant association was found between macrolide resistance and emm4, emm22 and emm77, whereas emm1, emm3, emm6, emm12, emm87 and emm89 were associated with macrolide susceptibility. A significant yearly fluctuation was observed in emm4, emm28 and emm77. The most common macrolide-resistant GAS were emm77 isolates harboring erm(A), either alone or in combination with mef(A), emm4 carrying mef(A), emm 28 possessing erm(B), emm75 carrying mef(A), emm 12 harboring mef(A) and emm 22 carrying erm(A). We estimated that $82.8 \%$ of the isolates belonged to emm types included in the novel 26 -valent $M$ protein vaccine. The vaccine coverage rate was determined mainly by the increased frequency of nonvaccine emm 4 isolates.
\end{abstract}

Conclusions: A limited number of emm types dominated among macrolide-susceptible and macrolide-resistant GAS isolates. We observed seasonal fluctuations, which were significant for emm4, emm 28 and emm77. This type of data can serve as baseline information if the novel 26-valent M protein GAS vaccine is introduced into practice.

Citation: Syrogiannopoulos GA, Grivea IN, Al-Lahham A, Panagiotou M, Tsantouli AG, et al. (2013) Seven-Year Surveillance of emm Types of Pediatric Group A Streptococcal Pharyngitis Isolates in Western Greece. PLoS ONE 8(8): e71558. doi:10.1371/journal.pone.0071558

Editor: Herminia de Lencastre, Rockefeller University, United States of America

Received January 7, 2013; Accepted July 1, 2013; Published August 19, 2013

Copyright: (c) 2013 Syrogiannopoulos et al. This is an open-access article distributed under the terms of the Creative Commons Attribution License, which permits unrestricted use, distribution, and reproduction in any medium, provided the original author and source are credited.

Funding: This study was supported by a grant from the German Ministry of Health (Bundesministerium für Gesundheit) and the 3089 grant from the Research Committee of the University of Thessaly. The funders had no role in study design, data collection and analysis, decision to publish, or preparation of the manuscript.

Competing Interests: The authors have read the journal's policy and have the following conflicts: MvdL has received research grants from Wyeth/Pfizer and GSK and acts as a consultant for Wyeth/Pfizer, Sanofi-Aventis and GSK. RRR is currently an employee of Pfizer Pharmaceuticals. ING, GAS, AA-L, MP and AGT declare no competing interests. There are no patents, products in development or marketed products to declare. This does not alter the authors' adherence to all the PLOS ONE policies on sharing data and materials, as detailed online in the guide for authors.

*E-mail: syrogian@otenet.gr

a Current address: Pfizer Pharmaceuticals, Paris, France

\section{Introduction}

Group A streptococcal (GAS) infections are a major cause of morbidity and mortality worldwide. GAS pharyngitis is one of the most common bacterial infections in school age children. Furthermore, GAS also causes a variety of skin and soft tissue infections, severe invasive disease, toxin-associated syndromes and the nonsuppurative sequelae of acute rheumatic fever, acute glomerulonephritis and, probably, reactive arthritis [1].
The M protein, encoded by the $\mathrm{emm}$ gene, is considered a major virulence factor and the major immunologic epitope of GAS [2]. The $\mathrm{M}$ protein possesses a hypervariable region of the aminoterminal 40 to 50 amino acid residues [3-6]. A GAS typing system based on sequencing of this N-terminal hypervariable region of the M protein $(\mathrm{emm})$ gene has been used for identification of different emm types. More than 150 types have been recognized worldwide to date $[7,8]$. 
Small N-terminal M protein peptides evoke protective antibodies against epidemiologically important GAS serotypes with the greatest bactericidal activity and are least likely to cross-react with human tissues. This discovery enabled investigators to develop recombinant multivalent $\mathrm{N}$-terminal type-specific vaccines $[9,10]$.

A multivalent vaccine encompassing small N-terminal M protein peptides from 26 different $\mathrm{emm}$ types has been developed and has entered clinical studies [11,12]. The effectiveness of this M protein vaccine may be highly dependent on the emm type coverage of the clinical isolates. And, therefore, the emm type distribution may guide further vaccine development.

The situation in Europe has drawn attention because of the significant variation in emm type distribution that might exist in relation to the increased rates of macrolide-resistant GAS isolates that have been noted in some countries in late $1990 \mathrm{~s}$ and early 2000 s [13-16]. Pharyngeal GAS emm type systematic surveillance enhances the understanding of the epidemiology of pharyngitis GAS disease and the formulation of multivalent type-specific vaccines.

Greece is a European country with increased rate of macrolideresistant GAS isolates $[17,18]$. We established a primary care network in Western Greece, in order to prospectively study children with acute GAS pharyngitis in a systematic fashion. The aim of the present 7-year study was to investigate among pharyngeal GAS isolates (i) the phenotypes and genotypes of macrolide-resistant isolates, (ii) the emm type and subtype distribution and (iii) the proportion of isolates that could be covered by the 26 -valent M protein-based GAS vaccine currently under clinical investigation.

\section{Materials and Methods}

\section{Ethics Statement}

The research protocol was approved by the Ethics Committee of the General University Hospital of Patras. A written informed consent was obtained from each child's parent or legal guardian. The data were analyzed anonymously.

\section{Subjects and specimens}

Between January 1999 and December 2005, 2408 GAS isolates were recovered from consecutive children with pharyngitis living in various areas of Western Greece. The study was conducted in 9 sites in a total of 5 prefectures: Preveza (Preveza), Agrinio and Nafpaktos (Etoloakarnania), Patras and Egion (Achaia), Pyrgos and Amaliada (Ilia), and Kalamata and Gargaliani (Messinia). During the 7-year study period, the estimated average population of Western Greece per study year was 950.306 inhabitants, which included 174.061 children aged $0-16$ years. The study population consisted of children 2-16 years old, with signs and symptoms of acute pharyngitis (fever, pharyngeal erythema and exudate, tender cervical lymph nodes, absence of conjunctivitis, rhinitis, hoarseness of voice or cough) confirmed by a positive throat culture for GAS. The study was performed in collaboration with 14 practicing pediatricians, who participate in our working group, the Hellenic Antibiotic-Resistant Respiratory Pathogens (HARP) Study Group. From November 2000 through December 2005, these pediatricians enrolled children with pharyngitis in clinical studies of different treatment regimens (Syrogiannopoulos GA, Grivea IN, Beratis NG, the HARP Study Group. $42^{\text {nd }}$ Intersci. Conf. Antimicrob. Agents Chemother., abstr. G-436, 2002; Syrogiannopoulos GA, Grivea IN, Kritikou D, the HARP Study Group. $43^{\text {rd }}$ Intersci. Conf. Antimicrob. Agents Chemother., abstr. G-1547, 2003; Syrogiannopoulos GA, Grivea IN, the HARP Study Group. $44^{\text {th }}$ Intersci. Conf. Antimicrob. Agents Chemother., abstr. G-
2092, 2004; Syrogiannopoulos GA, Grivea IN, Chryssanthopoulou DC, Katopodis GD, the HARP Study Group. $46^{\text {th }}$ Intersci. Conf. Antimicrob. Agents Chemother., abstr. G-842, 2006) [19]. One GAS isolate from each child was included in the study. Throat cultures were performed by the same investigator (ING) initially at the Laboratory of the Division of Pediatric Infectious Disease of the University of Patras (1999-2004) and subsequently at the Laboratory of the Division of Pediatric Infectious Disease of the University of Thessaly (2004-2005).

Isolates were identified as GAS by typical colony morphology, $\beta$-hemolysis on sheep blood agar, Lancefield grouping, by using a commercially available agglutination technique (Slidex, Streptokit; BioMérieux, Marcy l' Etoile, France), and by the pyrrolidonylarylamidase test.

GAS isolates were screened for susceptibility to erythromycin by both the disk diffusion method and the $\mathrm{E}$ test method (AB Biodisk, Sweden). The erythromycin-resistant GAS isolates were further studied for their antimicrobial susceptibility to erythromycin or clarithromycin by the broth microdilution method as described previously [17].

Over the 7-year study period, resistance to macrolides was found in $548(22.8 \%)$ of the 2408 GAS isolates.

\section{Determination of macrolide resistance phenotypes}

The macrolide resistance phenotypes, i.e. M, inducible MLS (iMLS) and constitutive MLS (cMLS), as well as their subtypes [20], were determined on the basis of the pattern of susceptibility to erythromycin and clindamycin and confirmed by the triple-disk (erythromycin plus clindamycin and josamycin) test. The tripledisk test was set up to facilitate the laboratory discrimination of the 3 subtypes (iMLS-A, iMLS-B, and iMLS-C) of the iMLS macrolide resistance phenotype, as described previously [20].

\section{Macrolide resistance determinants}

GAS isolates showing resistance to erythromycin were tested by PCR for the presence of $\operatorname{erm}(\mathrm{A}), \operatorname{erm}(\mathrm{B})$, or mef(A) macrolide resistance determinants [21].

\section{emm Typed sample of macrolide-resistant GAS isolates}

A representative sample of $338(61.7 \%)$ of the 548 macrolideresistant isolates was studied for their emm type and the presence of macrolide resistance determinants. The sample consisted of 181 $(47.8 \%)$ of the 379 macrolide-resistant isolates collected during 1999-2002 and 157 (92.9\%) of the 169 macrolide-resistant isolates from 2003-2005. For 1999-2002, the random selection was stratified by year taking into account the yearly number of isolates, the proportions of the different resistance phenotypes, the season and the study sites.

\section{emm Typed sample of macrolide-susceptible GAS isolates}

Two hundred and five (11\%) of the total 1860 macrolidesusceptible isolates were analyzed for their $\mathrm{emm}$ type. The random selection was stratified by year taking into account the yearly number of isolates, the season and the study site.

The isolates were studied for their emm type according to the method of Podbielski et al. [22]. Similarity searching was performed by using the N-terminal hypervariable region of the $\mathrm{M}$ gene based on the latest information from the Centers for Disease Control website (cdc.gov/ncidod/-biotech/strep/strains/ emmtypes.html). GAS CS101 (emm49) was used as a reference strain. A limited number of isolates from 1999-2002 was emm typed and published previously [17]. 


\section{Statistical analysis}

In each isolate a number was assigned and then, the numbers were entered into an array. Thereafter a CVF90 subtractive with a random generator number was applied to the array to select the isolates for emm type analysis. For the assessment of 2 groups, categorical parameters were compared using the Fisher's exact test. We independently assessed the difference between susceptible versus resistant isolates for a given $\mathrm{emm}$ type, thus we did not adjust for multiplicity [23]. Yearly fluctuation was tested using a loglinear model, with the year effect as exploratory variable and as response either the frequency of a certain $\mathrm{emm}$ type or the rate of macrolide-resistant isolates. Vaccine coverage was defined as the proportion of all isolates in the region that were covered by the 26valent $M$ protein-based GAS vaccine currently under clinical investigation [11]. The overall vaccine coverage was calculated as the weighted average of the two vaccine coverage rates: the one corresponding to the susceptible isolates and the one corresponding to the resistant isolates. We compared the vaccine coverage of the first versus the second period using z-test. Two-sided tests were used. The statistical analysis was performed using SPSS version 13.0 (SPSS Inc., Chicago, Ill). An effect was considered significant when $P<0.05$.

\section{Results}

\section{Pharyngeal GAS isolates}

During the 7-year study period, 2408 GAS isolates were recovered from children with pharyngitis. GAS infections were mainly seen among children 5 to 10 years of age (79.4\% of cases); the median age was 7 years. The number of GAS isolates gathered yearly, the rate of macrolide resistance and the resistance phenotypes appear in Figure 1. Resistance to macrolides was found in $548(22.8 \%)$ of the 2408 isolates. More than half $(52.6 \%)$ of these macrolide-resistant isolates exhibited either the inducible or the constitutive MLS phenotype. Specifically, $47.4 \%$ had the M phenotype, $0.7 \%$ the iMLS-A, $18.3 \%$ had the iMLS-B, $26.5 \%$ the iMLS-C, and finally $7.1 \%$ had the cMLS phenotype.

Along the study period we noted a tendency towards significantly decreased rates of resistance $(P<0.001)$. The lowest rates occurred in 2002 (15.3\%), 2003 (15\%) and 2004 (16.7\%). In 2005 , an increase of isolates exhibiting the iMLS phenotype was

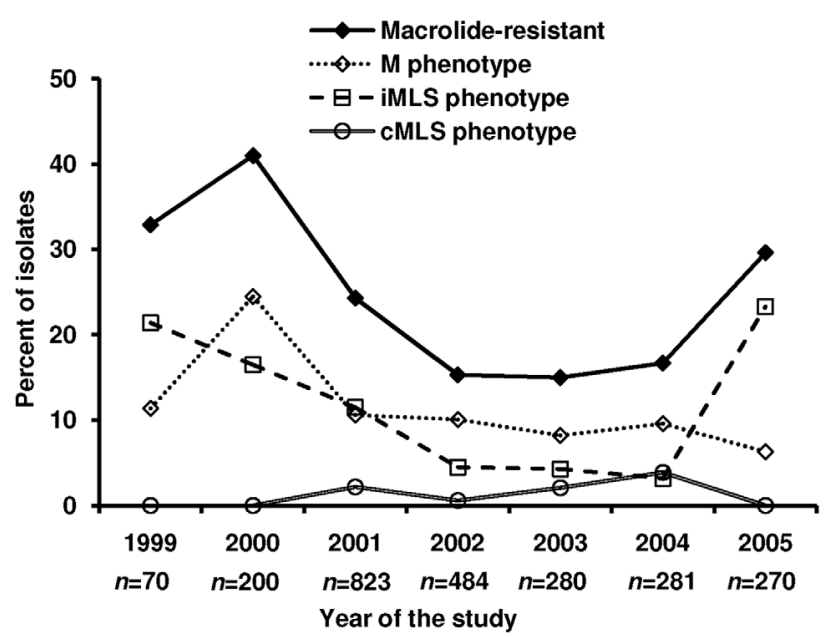

Figure 1. Macrolide resistance rate and resistance phenotypes among $\mathbf{2 4 0 8}$ group A streptococcal isolates in Western Greece. doi:10.1371/journal.pone.0071558.g001 noted; $92 \%$ had the iMLS-B subtype, $4.8 \%$ the iMLS-C and 3.2\% the iMLS-A.

A random sample of 543 isolates, 205 macrolide-susceptible and 338 macrolide-resistant, was further analyzed with resistance genotyping and emm typing. Of the 338 macrolide-resistant isolates, $43.5 \%$ had the M phenotype, $1.2 \%$ the iMLS-A, $23.1 \%$ showed the iMLS-B, $21.6 \%$ the iMLS-C, and finally $10.6 \%$ had the cMLS phenotype. There was no significant difference in the frequency of any given phenotype in the typed sample of macrolide-resistant isolates compared to that found in the total collection of macrolide-resistant isolates. The 338 typed macrolide-resistant isolates harbored the mef(A) $(43.7 \%)$, erm $(\mathrm{A})(36.1 \%)$, erm(A) plus mef(A) $(8.9 \%), \operatorname{erm}(\mathrm{B})(8.6 \%)$ or $\mathrm{erm}(\mathrm{B})$ plus mef(A) $(2.7 \%)$ gene. A combination of macrolide resistance determinants was revealed in $11.5 \%$ of macrolide-resistant isolates.

\section{emm Types of GAS isolates}

The 543 typed isolates belonged to 28 different emm types; the macrolide-susceptible belonged to 25 types and the macrolideresistant to 18 (Table 1). A statistically significant association was found between macrolide resistance and emm4, emm 22 and $е m m 77$, whereas emm1, emm3, етm6, етm12, етm87 and emm89 were associated with macrolide susceptibility. However, there were 2 distinct groups of isolates regarding their association with macrolide resistance or susceptibility. In the one group of emm types, emm4, emm12, emm22 and emm77, were observed populations of both macrolide-resistant and -susceptible isolates. In contrast, in the other group of emm types, emm3, emm 6 and emm87, macrolideresistant isolates were rare.

Among the macrolide-susceptible isolates, $4 \mathrm{emm}$ types, emm 12 , emm 1 , emm 77 and emm 89 , accounted for $51.7 \%$ of isolates, while 7 types, етm12, етm1, етm77, етm89, етm 3, етm6 and етm28,

Table 1. emm type distribution of the macrolide-susceptible and macrolide-resistant group A streptococcal isolates.

\begin{tabular}{|c|c|c|c|}
\hline \multirow{2}{*}{ emm type } & \multirow{2}{*}{$\begin{array}{l}\begin{array}{l}\text { Macrolide- } \\
\text { susceptible }\end{array} \\
(n=205)\end{array}$} & \multirow{2}{*}{$\begin{array}{l}\begin{array}{l}\text { Macrolide- } \\
\text { resistant }\end{array} \\
(n=338)\end{array}$} & \multirow[t]{2}{*}{$P$} \\
\hline & & & \\
\hline 12 & $44(21.5)^{\mathrm{a}}$ & $25(7.4)$ & $<0.001$ \\
\hline 1 & $28(13.7)$ & $9(2.7)$ & $<0.001$ \\
\hline 77 & $19(9.3)$ & 100 (29.6) & $<0.001$ \\
\hline 89 & $15(7.3)$ & $11(3.3)$ & 0.038 \\
\hline 3 & $13(6.3)$ & $1(0.3)$ & $<0.001$ \\
\hline 6 & $13(6.3)$ & $2(0.6)$ & $<0.001$ \\
\hline 28 & $13(6.3)$ & $26(7.7)$ & 0.610 \\
\hline 2 & $11(5.4)$ & $11(3.3)$ & 0.264 \\
\hline 4 & $10(4.9)$ & $98(29)$ & $<0.001$ \\
\hline 11 & $10(4.9)$ & $9(2.7)$ & 0.228 \\
\hline 75 & $7(3.4)$ & $19(5.6)$ & 0.302 \\
\hline 87 & $5(2.4)$ & $1(0.3)$ & 0.031 \\
\hline 22 & $3(1.5)$ & $21(6.2)$ & 0.009 \\
\hline Other & $14^{\mathrm{b}}(6.8)$ & $5^{c}(1.5)$ & 0.001 \\
\hline
\end{tabular}

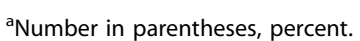

bemm type (no. of isolates): 23 (1), 25 (1), 29 (2), 44 (1), 50 (1), 65 (1), 68 (1), 102 (1), 110 (2), 118 (1), PT3875 (1), ST3211 (1).

cemm type (no. of isolates): 15 (1), 25 (1), 49 (1), 102 (1), 106 (1).

doi:10.1371/journal.pone.0071558.t001 
accounted for $70.7 \%$ and the 10 most prevalent for $85.9 \%$ of isolates (Table 1).

Macrolide-resistant isolates belonged to a limited number of emm types. Specifically, emm 4 , emm 75 , emm 12 and emm 1 accounted for $72.3 \%$ of the erythromycin-resistant GAS isolates harboring mef(A) as the sole macrolide resistance determinant (Table 2). Furthermore, emm 77 , emm 4 and emm 22 accounted for $77 \%$ of those carrying erm(A), either alone or in combination with mef(A), whereas $\mathrm{emm} 28$ and $\mathrm{emm} 12$ accounted for $57.9 \%$ of the possessing $\operatorname{erm}(\mathrm{B})$ isolates, either alone or in combination with mef(A).

There was seasonal fluctuation in the predominant $\mathrm{emm}$ types. Among macrolide-susceptible isolates, the variation in those belonging to emm 28 and $\mathrm{emm} 77$ reached a statistically significant level $(P<0.01)$. On the other hand, among macrolide-resistant isolates, a significant seasonal fluctuation was observed in those belonging to emm4, emm 28 and $\mathrm{emm} 77$ (Figure 2). In 2005, we noted an increased number of GAS isolates belonging to emm77, expressing the iMLS phenotype. They all exhibited the iMLS-B subtype and harboured $\operatorname{erm}(\mathrm{A})$, either alone or in combination with $\operatorname{mef}(\mathrm{A})$.

Prevalence of the classic rheumatogenic types (emm3, emm5, emm6, emm14, emm18, emm19 and emm29)

Of the 543 typed pharyngeal isolates, only two belonged to emm 29 and none to emm5, emm 14, emm 18, and emm19. In addition, emm 3 and emm 6 made up to $6.3 \%$ of macrolide-susceptible isolates each, while they represented $0.3 \%$ and $0.6 \%$ of macrolideresistant isolates, respectively.

\section{Subtypes}

Among the 10 most prevalent emm types, we observed considerable variability in the frequencies of the respective subtypes. We identified $12 \mathrm{emm}$ subtypes: emm1.14, етm 1.37, eтm3.1, eтm3.35, emm6.4, emm6.54, emm12.11, emm12.21, eтm12.23, етm12.37, етm28.5, етm 29.4. Within emm1, emm3, emm6, етm 12, етm 28 and emm 29 isolates, there were 1-4 subtypes, accounting for $2.6 \%-50 \%$ of all isolates of a given $\mathrm{emm}$ type.

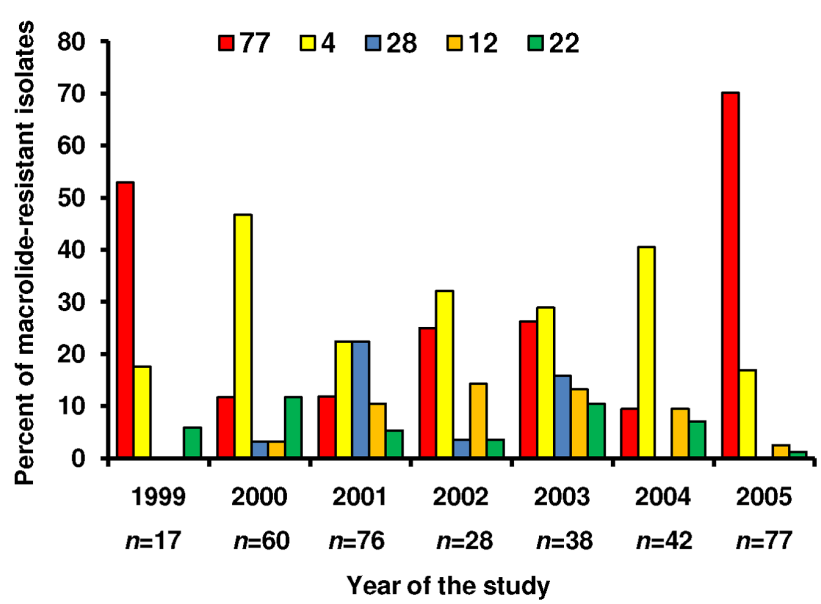

Figure 2. Macrolide-resistant group A streptococcal isolates. Annual frequencies of the 5 most common emm types. A significant yearly variation was observed in emm28 $(P<0.001)$, emm77 $(P<0.001)$ and emm4 $(P<0.005)$.

doi:10.1371/journal.pone.0071558.g002

emm Types of the representative isolates in relation to an experimental 26-valent GAS vaccine

Twelve of the $28 \mathrm{emm}$ types found in Greek pharyngeal isolates are included in an experimental 26-valent GAS vaccine. The cumulative distributions of emm types of macrolide-susceptible and macrolide-resistant isolates are presented in Figure 3. We found that $178(86.8 \%)$ of the 205 macrolide-susceptible isolates and 234 $(69.2 \%)$ of the 338 macrolide-resistant isolates belonged to $\mathrm{emm}$ types that are included in the vaccine $(P<0.001)$. The lower coverage of macrolide-resistant isolates was attributed to 7 nonvaccine emm types, emm4 (predominant), emm15, emm25, emm49, emm87, emm102 and emm106.

Among macrolide-susceptible isolates, the vaccine coverage was 84.9\% for $1999-2002$ vs. $90.8 \%$ for $2003-2005$ (z-test $=1.204$, $P=0.277)$. On the other hand, among macrolide-resistant isolates,

Table 2. emm type distribution of the 338 macrolide-resistant group A streptococcal isolates with different macrolide resistance genotypes.

\begin{tabular}{|c|c|c|c|c|c|c|}
\hline \multirow[b]{2}{*}{ emm type } & \multirow[b]{2}{*}{ No. of isolates } & \multicolumn{5}{|c|}{ Macrolide resistance genotypes } \\
\hline & & $\operatorname{mef}(\mathbf{A})$ & $\operatorname{erm}(\mathbf{A})$ & $\operatorname{erm}(\mathbf{A})$ plus mef(A) & erm(B) & erm(B) plus mef(A) \\
\hline 77 & 100 & $13(13)^{a}$ & $62(62)$ & $24(24)$ & 0 & $1(1)$ \\
\hline 4 & 98 & $74(75.5)$ & $13(13.3)$ & $5(5.1)$ & $2(2)$ & $4(4.1)$ \\
\hline 28 & 26 & $7(26.9)$ & $3(11.5)$ & 0 & $16(61.5)$ & 0 \\
\hline 12 & 25 & $13(52)$ & $6(24)$ & 0 & $5(20)$ & $1(4)$ \\
\hline 22 & 21 & $6(28.6)$ & $13(61.9)$ & 0 & $1(4.8)$ & $1(4.8)$ \\
\hline 75 & 19 & $16(84.2)$ & $2(10.5)$ & 0 & $1(5.3)$ & 0 \\
\hline 2 & 11 & 0 & $11(100)$ & 0 & 0 & 0 \\
\hline 89 & 11 & $5(45.5)$ & $3(27.3)$ & 0 & $2(18.2)$ & $1(9.1)$ \\
\hline 1 & 9 & $4(44.4)$ & $4(44.4)$ & 0 & 0 & 1 (11.1) \\
\hline 11 & 9 & $5(55.6)$ & $2(22.2)$ & 0 & $2(22.2)$ & 0 \\
\hline Other & 9 & $5^{b}(55.6)$ & $3^{c}(33.3)$ & $1^{d}(11.1)$ & 0 & 0 \\
\hline
\end{tabular}

${ }^{a}$ Number in parentheses, percent.

bemm type (no. of isolates): 3 (1), 6 (1), 49 (1), 102 (1), 106 (1).

cemm type (no. of isolates): 6 (1), 15 (1), 87 (1).

demm type (no. of isolates): 25 (1).

doi:10.1371/journal.pone.0071558.t002 


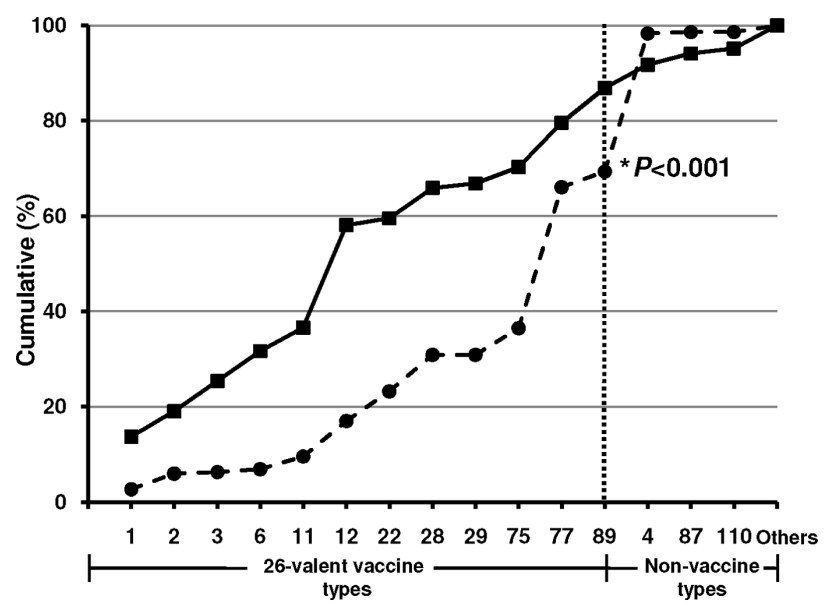

Figure 3. Cumulative distribution of the emm types of the macrolide-susceptible (solid line) and the macrolide-resistant (dashed line) group A streptococcal (GAS) isolates, according to the types included in an experimental 26-valent GAS vaccine.

doi:10.1371/journal.pone.0071558.g003

the vaccine coverage was $65.7 \%$ for $1999-2002$ vs. $73.2 \%$ for 2003-2005 (z-test $=1.5, P=0.157)$.

\section{Estimated overall coverage rate by an experimental 26- valent $M$ protein GAS vaccine}

We applied the coverage rate observed among the representative macrolide-susceptible isolates and the one found among the representative macrolide-resistant isolates in order to estimate the overall coverage rate in the total collection of macrolidesusceptible and macrolide-resistant isolates gathered over the 7year study.

Total number of macrolide-susceptible isolates x coverage rate among macrolide-susceptible isolates $(\%)+$ total number of macrolide-resistant isolates $\mathrm{x}$ coverage rate among macrolideresistant isolates $(\%) /$ total number of isolates.

\section{Discussion}

We assessed temporal trends in GAS emm type distribution among pediatric pharyngeal isolates collected systematically from 9 scattered geographic sites in Western Greece over a 7-year period. Special attention has been paid to macrolide-resistant isolates. This is one of the largest published collections of emm typed macrolide-resistant isolates along with a random sample of macrolide-susceptible isolates recovered from children with pharyngitis. Several features are of importance in this prospective study: number of patients enrolled (over 2400), duration of the observation (7 years), homogeneity of patients enrolled in terms of age (2-16 years) and of clinical presentation, same investigators and same methodology throughout the study period.

In Greece from 1990 to 2005, macrolide and lincosamide consumption has been substantially increased, with the highest consumption rate noted in 2004 (2005 IMS Health database) [24]. Among GAS isolates circulating in Western Greece, there was an initial phase of a prominent increase in the rate of macrolide resistance, 1999-2000, followed by a second phase, initiated in 2001, characterized by a significant trend of decline in the resistance rate. Increased rates of macrolide-resistant GAS, sometimes as high as $35 \%$, followed by a decrease have been also reported in recent studies from other European countries $[25,26]$. It has been suggested that this decline may be attributed to decreased macrolide consumption [27] as well as GAS clonal properties [28]. Even though there has been a decrease in the rates of macrolide resistance among GAS isolates, it should be underlined that Greece remains one of the countries with the highest macrolide resistance rates in Europe. Specifically, the rate of macrolide-resistant GAS isolates ranged from $18.8 \%$ in Athens, Greece, during 2006 [29] to $24 \%$ in Central Greece, between January 2007 and June 2009 [18].

In this study, a statistically significant association was found between macrolide susceptibility and emm1, emm3, етm6, етm12, emm 87 and emm89. In previous reports, a similar correlation was found between macrolide susceptibility and emm 1 [29,30], emm3 [28,30], emm 12 [30], emm6 [28] and emm89 [28]. On the other hand, we also established significant association between macrolide resistance and $\mathrm{emm} 4$, emm 22 and $\mathrm{emm} 77$. In previous studies, a similar correlation has been found for emm 4 [28,30-32], emm22 [28,29,31] and emm77 [29].

Although we observed considerable diversity in emm types among macrolide-susceptible GAS isolates, relatively few types dominated. The 7 most prominent emm types were emm12, emm l, етm77, етm89, етm3, етm 6 and emm28 (in descending order) and accounted for $70.7 \%$ of susceptible isolates. Similarly, in a 7-year surveillance of pediatric pharyngitis isolates from North America, these $7 \mathrm{emm}$ types were among the 10 most common emm types in the United States, accounting for $67.1 \%$ of isolates, and among the 9 most common $\mathrm{emm}$ types in Canada, accounting for $68.6 \%$ of isolates [33]. In this large surveillance, the prevalence was estimated on the basis of the total number of isolates and not according to macrolide susceptibility. It should be underlined that the macrolide resistance rates in these countries are significantly lower compared to those observed in our study [34]. Furthermore, a similar dominance by relatively few types has been reported in studies of pharyngeal isolates from Ontario, Canada [35] and Western European countries, such as Sweden [36].

The present study showed that the majority of macrolideresistant isolates belonged to a limited number of emm types. emm4, emm 75 , emm 12, and emm 1 accounted for $72.3 \%$ of the erythromycin-resistant GAS isolates harboring mef(A) as the sole macrolide resistance determinant. The same types accounted for $68.2 \%$, $77.1 \%$, and $100 \%$ of mef(A)-positive macrolide-resistant GAS isolates in Italy, North America, and France, respectively [13,34,37]. erm(A), either alone or in combination with mef(A), was carried mainly by emm 77 isolates. The same type accounted for $100 \%$ of GAS isolates possessing $\mathrm{erm}(\mathrm{A})$ in an study in Italy [37]. Moreover, Zampaloni et al. [31] studied the macrolide resistance phenotypes, including subtypes, and reported that 12 $(92.3 \%)$ of the 13 isolates exhibiting the iMLS-B or iMLS-C belonged to emm 77. Finally, in the present study, emm 28 was found to be predominant among the $\mathrm{erm}(\mathrm{B})$-positive macrolide-resistant GAS isolates. The same type accounted for $50 \%$ and $70 \%$ of the $\operatorname{erm}(\mathrm{B})$-positive macrolide-resistant GAS isolates in the United States and France, respectively [13,34].

Our 7-year survey revealed a marked prevalence of macrolideresistant GAS isolates belonging to emm 4 . The frequency of emm 4 was 5.9-fold higher among macrolide-resistant GAS compared to macrolide-susceptible isolates. Increased prevalence of emm 4 has been identified in a recent survey from Ontario, Canada [35] as well as in other pharyngitis surveys $[28,33,36]$. A high frequency of emm4 among macrolide-resistant GAS isolates, mostly with $\mathrm{M}$ phenotype, has been described in Spain (ranked $1^{\text {st }}$ ) [30], in Italy $\left(2^{\text {nd }}\right.$ marginally) [31], in Portugal $\left(2^{\text {nd }}\right)$ [28] and in the United States $\left(3^{\text {rd }}\right)[34]$. 
emm 77 was ranked third among our macrolide-susceptible GAS isolates and first among macrolide-resistant. However, it was found to have a rather low frequency in a thorough review of the available global data on GAS epidemiology [38].

This survey clearly demonstrates fluctuation in the frequency of pharyngeal emm types from season to season in Western Greece. A significant fluctuation was observed in emm 4 , emm 28 and $e m m 77$. A recent study from Canada reported yearly fluctuations for $\mathrm{emm} 1$, emm 3 , emm 28 and emm 77 [35]. It has been postulated that the increased level of immunity to certain predominant types contributes to the decreased prevalence of some of these types in subsequent years [33]. Furthermore, it has been reported that several of the most common emm types account for a progressively lower proportion of pharyngeal isolates among older children and adolescents compared to younger children, suggesting a role for type-specific immunity [39]. In addition, it has been reported that serum antibody levels to several common $\mathrm{M}$ proteins were higher in older than in younger children [40].

Our data show that the classic rheumatogenic types had decreased prevalence, and in some instances completely disappeared. This decrease parallels the marked decline in incidence of acute rheumatic fever in our country [41].

In Western Greece during the study period, the 26-valent GAS vaccine coverage rate was estimated to be $82.8 \%$; $\mathrm{emm} 4$ was the most common nonvaccine type. This coverage rate is close to that reported in recent studies on pharyngitis from North America. In a recent study of pediatric pharyngitis from the USA and Canada, the 26-valent vaccine coverage was approximately $85 \%$ [33]. In a study from Ontario, Canada, the 26 -valent GAS vaccine coverage was $78.5 \%$ for pharyngitis cases, mean age 16.1 years, range 8 months to 105 years [35]. The vaccine coverage is higher in GAS pharyngitis rather than in invasive disease, which represents a major target for GAS immunization [42,43]. Furthermore, it is higher in Established Market Economy countries than in lower income countries [38]. Lower coverage rates have been noted in other parts of the world, such as New Zealand [44].

It is thought that the circulation of macrolide-resistant isolates belonging to a common emm type, such as $\mathrm{emm} 4$, may be enhanced if a vaccine not including this type is used. emm 4 has been included in a new 30-valent $M$ protein-based GAS vaccine, which has entered the pre-clinical phase of evaluation [45].

\section{References}

1. Stevens DL (2000) Group A beta-hemolytic streptococci: virulence factors, pathogenesis, and spectrum of clinical infections. In: Stevens DL, Kaplan EL, editors. Streptococcal infections: clinical aspects, microbiology, and molecular pathogenesis. New York: Oxford University Press. 19-36.

2. Lancefield RC (1962) Current knowledge of type-specific M antigens of group A streptococci. J Immunol 89: 307-313.

3. Beachey EH, Seyer JM, Dale JB, Simpson WA, Kang AH (1981) Type-specific protective immunity evoked by synthetic peptide of Streptococcus pyogenes M protein. Nature (London) 292: 457-459.

4. Jones KF, Manjula BN, Johnston KH, Hollingshead SK, Scott JR, et al. (1985) Location of variable and conserved epitopes among the multiple serotypes of streptococcal M protein. J Exp Med 161: 623-628.

5. Dale JB, Beachey EH (1986) Localization of protective epitopes of the amino terminus of type 5 streptococcal M protein. J Exp Med 163: 1191-1202.

6. Beall B, Facklam R, Thompson T (1996) Sequencing emm-specific PCR products for routine and accurate typing of group A streptococci. J Clin Microbiol 34: 953-958.

7. Bisno AL, Brito MO, Collins CM (2003) Molecular basis of group A streptococcal virulence. Lancet Infect Dis 3: 191-200.

8. Cohen-Poradosu R, Kasper DL (2007) Group A streptococcus epidemiology and vaccine implications. Clin Infect Dis 45: 863-865.

9. Dale JB, Simmons M, Chiang EC, Chiang EY (1996) Recombinant, octavalent group A streptococcal M protein vaccine. Vaccine 14: 944-948.

10. Dale JB (1999) Multivalent group A streptococcal vaccine designed to optimize the immunogenicity of six tandem M protein fragments. Vaccine 17: 193-200.
A limitation of the present study may be the fact that we did not emm type a larger number or even the total number of macrolidesusceptible isolates. However, when we evaluated the typed macrolide-susceptible and macrolide-resistant isolates according to two time-periods, i.e. 1999-2002 compared to 2003-2005, we did not find a significant difference in their respective vaccine coverage rates.

In conclusion, a limited number of emm types dominated among macrolide-susceptible and macrolide-resistant GAS isolates recovered from consecutive children with pharyngitis in Western Greece during 1999-2005. A statistically significant association was found between macrolide resistance and emm4, emm22 and етm77, whereas emm1, етm3, етm6, етm12, етm87 and етm89 were associated with macrolide susceptibility. We observed seasonal fluctuations, which were significant for emm 4, emm 28 and emm 77 . We estimated that $82.8 \%$ of the pharyngeal isolates belonged to emm types that are included in an experimental 26-valent $\mathrm{M}$ protein vaccine. emm 4 was the most common nonvaccine type. The molecular epidemiology data on macrolide-resistant and macrolide-susceptible GAS isolates in different time-periods and various areas, especially those with high prevalence of macrolideresistant GAS, can serve as baseline information if the novel 26valent $\mathrm{M}$ protein GAS vaccine is introduced into practice.

\section{Acknowledgments}

We acknowledge the investigators who enrolled patients: Garyfalia Chioti, Kalamata; Georgia Danasi-Loukopoulou, Nafpaktos; Panagiotis Fakos, Patras; Ekaterini Ifanti, Egion; Anthi Kakogianni, Patras; Kyriaki Kotsioni, Amaliada; Elias Mamalis, Agrinio; Christos Mitselos, Pyrgos; Constantinos Panagopoulos, Patras; Dimitrios Papalabrou, Patras; Irini Papanagiotou, Agrinio; Eugenia Passa, Preveza; Ioannis Psaras, Egion; and Alexandros Tzortzinis, Gargaliani. We also acknowledge Dr. Elias Zintzaras for performing the statistical analysis, Nelli Neuberger for excellent technical assistance and Dr. Denise C. Chryssanthopoulou for critically reviewing the manuscript.

\section{Author Contributions}

Conceived and designed the experiments: GAS ING RRR MvdL. Performed the experiments: ING AA-L MP. Analyzed the data: MP AGT ANM. Contributed reagents/materials/analysis tools: GAS RRR MvdL. Wrote the paper: GAS ING AA-L MP AGT ANM RRR MvdL.

11. Hu MC, Walls MA, Stroop SD, Reddish MA, Beall B, et al. (2002) Immunogenicity of a 26 -valent group A streptococcal vaccine. Infect Immun 70: 2171-2177.

12. McNeil SA, Halperin SA, Langley JM, Smith B, Warren A, et al. (2005) Safety and immunogenicity of 26-valent group A streptococcus vaccine in healthy adult volunteers. Clin Infect Dis 41: 1114-1122.

13. Bingen E, Bidet P, Mihaila-Amrouche L, Doit C, Forcet S, et al. (2004) Emergence of macrolide-resistant Streptococcus pyogenes strains in French children. Antimicrob Agents Chemother 48: 3559-3562.

14. Silva-Costa C, Ramirez M, Melo-Cristino J, the Portuguese Surveillance Group for the Study of Respiratory Pathogens (2005) Rapid inversion of the prevalences of macrolide resistance phenotypes paralleled by a diversification of $\mathrm{T}$ and $\mathrm{emm}$ types among Streptococcus pyogenes in Portugal. Antimicrob Agents Chemother 49: 2109-2111.

15. Mazzariol A, Koncan R, Bahar G, Cornaglia G (2007) Susceptibilities of Streptococcus pyogenes and Streptococcus pneumoniae to macrolides and telithromycin: data from an Italian multicenter study. J Chemother 19: 500-507.

16. Rubio-López V, Valdezate S, Álvarez D, Villalón P, Medina MJ, et al. (2012) Molecular epidemiology, antimicrobial susceptibilities and resistance mechanisms of Streptococcus pyogenes isolates resistant to erythromycin and tetracycline in Spain (1994-2006). BMC Microbiol 12: 215.

17. Grivea IN, Al-Lahham A, Katopodis GD, Syrogiannopoulos GA, Reinert RR (2006) Resistance to erythromycin and telithromycin in Streptococcus pyogenes isolates obtained between 1999 and 2002 from Greek children with tonsillopharyngitis: phenotypic and genotypic analysis. Antimicrob Agents Chemother 50: 256-261. 
18. Malli E, Tatsidou E, Damani A, Pantelidi K, Petinaki E, et al. (2010) Macrolideresistant Streptococcus pyogenes in Central Greece: prevalence; mechanism and molecular identification. Int J Antimicrob Agents 35: 614-615.

19. Syrogiannopoulos GA, Bozdogan B, Grivea IN, Ednie LM, Kritikou DI, et al. (2004) Two dosages of clarithromycin for five days, amoxicillin/clavulanate for five days or penicillin $\mathrm{V}$ for ten days in acute group $\mathrm{A}$ streptococcal tonsillopharyngitis. Pediatr Infect Dis J 23: 857-865.

20. Giovanetti E, Montanari MP, Mingoia M, Varaldo PE (1999) Phenotypes and genotypes of erythromycin-resistant Streptococcus pyogenes strains in Italy and heterogeneity of inducibly resistant strains. Antimicrob Agents Chemother 43: 1935-1940.

21. Brandt CM, Honscha M, Truong ND, Holland R, Hövener B, et al. (2001) Macrolide resistance in Streptococcus pyogenes isolates from throat infections in the region of Aachen, Germany. Microb Drug Resist 7: 165-170.

22. Podbielski A, Melzer B, Lütticken R (1991) Application of the polymerase chain reaction to study the $\mathrm{M}$ protein (-like) gene family in beta-hemolytic streptococci. Med Microbiol Immunol 180: 213-227.

23. Rothman KJ (1990) No adjustments are needed for multiple comparisons. Epidemiology 1: 43-46.

24. Daikos GL, Koutsolioutsou A, Tsiodras S, Theodoridou M, Koutouzis EI, et al. (2008) Evolution of macrolide resistance in Streptococcus pneumoniae clinical isolates in the prevaccine era. Diagn Microbiol Infect Dis 60: 393-398.

25. Silva-Costa C, Pinto FR, Ramirez M, Melo-Cristino, the Portuguese Surveillance Group for the Study of Respiratory Pathogens (2008) Decrease in macrolide resistance and clonal instability among Streptococcus pyogenes in Portugal. Clin Microbiol Infect 14: 1152-1159.

26. Ardanuy C, Domenech A, Rolo D, Calatayud L, Tubau F, et al. (2010) Molecular characterization of macrolide- and multidrug-resistant Streptococcus pyogenes isolated from adult patients in Barcelona, Spain (1993-2008). J Antimicrob Chemother 65: 634-643.

27. Van Heirstraeten L, Coenen S, Lammens C, Hens N, Goossens H, et al. (2012) Antimicrobial drug use and macrolide-resistant Streptococcus pyogenes, Belgium. Emerg Infect Dis 18: 1515-1518.

28. Silva-Costa C, Friães A, Ramirez M, Melo-Cristino, the Portuguese Group for the Study of Streptococcal Infections (2012) Differences between macrolideresistant and -susceptible Streptococcus pyogenes: importance of clonal properties in addition to antibiotic consumption. Antimicrob Agents Chemother 56: 56615666 .

29. Michos AG, Bakoula CG, Braoudaki M, Koutouzi FI, Roma ES, et al. (2009) Macrolide resistance in Streptococcus pyogenes: prevalence, resistance determinants, and emm types. Diagn Microbiol Infect Dis 64: 295-99.

30. Albertí S, García-Rey C, Domínguez MA, Aguilar L, Cercenado E, et al. (2003) Survey of emm gene sequences from pharyngeal Streptococcus pyogenes isolates collected in Spain and their relationship with erythromycin susceptibility. J Clin Microbiol 41: 2385-2390.
31. Zampaloni C, Cappelletti P, Prenna M, Vitali LA, Ripa S (2003) emm Gene distribution among erythromycin-resistant and -susceptible Italian isolates of Streptococcus pyogenes. J Clin Microbiol 41: 1307-10.

32. Rivera A, Rebollo M, Miró E, Mateo M, Navarro F, et al. (2006) Superantigen gene profile, emm type and antibiotic resistance genes among group A streptococcal isolates from Barcelona, Spain. J Med Microbiol 55: 1115-1123.

33. Shulman ST, Tanz RR, Dale JB, Beall B, Kabat W, et al. (2009) Seven-year surveillance of North American pediatric group A streptococcal pharyngitis isolates. Clin Infect Dis 49: 78-84.

34. Tanz RR, Shulman ST, Shortridge VD, Kabat W, Kabat K, et al. (2004) Community-based surveillance in the United States of macrolide-resistant pediatric pharyngeal group A streptococci during 3 respiratory disease seasons. Clin Infect Dis 39: 1794-1801.

35. Shea PR, Ewbank AL, Gonzalez-Lugo JH, Martagon-Rosado AJ, MartinezGutierrez JC, et al. (2011) Group A Streptococcus emm gene types in pharyngeal isolates, Ontario, Canada, 2002-2010. Emerg Infect Dis 17: 2010-2017.

36. Darenberg J, Luca-Harari B, Jasir A, Sandgren A, Pettersson H, et al. (2007) Molecular and clinical characteristics of invasive group A streptococcal infection in Sweden. Clin Infect Dis 45: 450-458.

37. Dicuonzo G, Fiscarelli E, Gherardi G, Lorino G, Battistoni F, et al. (2002) Erythromycin-resistant pharyngeal isolates of Streptococcus pyogenes recovered in Italy. Antimicrob Agents Chemother 46: 3987-3990.

38. Steer AC, Law I, Matatolu L, Beall BW, Carapetis JR (2009) Global emm type distribution of group A streptococci: systematic review and implications for vaccine development. Lancet Infect Dis 9: 611-616.

39. Jaggi P, Tanz RR, Beall B, Shulman ST (2005) Age influences the emm type distribution of pediatric group A streptococcal pharyngeal isolates. Pediatr Infect Dis J 24: 1089-1092.

40. Jaggi P, Dale JB, Chiang E, Beniwal P, Kabat W, et al. (2009) Age-associated differences in prevalence of group A streptococcal type-specific $\mathrm{M}$ antibodies in children. Eur J Pediatr 168: 679-683.

41. Kafetzis DA, Chantzi FM, Grigoriadou G, Vougiouka O, Liapi G (2005) Incidence and clinical profile of acute rheumatic fever in Greece. Eur J Clin Microbiol Infect Dis 24: 68-70.

42. O'Loughlin RE, Roberson A, Cieslak PR, Lynfield R, Gershman K, et al, (2007) The epidemiology of invasive group A streptococcal infection and possible vaccine implications: United States, 2000-2004. Clin Infect Dis 45: 853-862.

43. Luca-Harari B, Darenberg J, Neal S, Siljander T, Strakova L, et al. (2009) Clinical and microbiological characteristics of severe Streptococcus pyogenes disease in Europe. J Clin Microbiol 47: 1155-1165.

44. Safar A, Lennon D, Stewart J, Trenholme A, Drinkovic D, et al. (2011) Invasive group A streptococcal infection and vaccine implications, Auckland, New Zealand. Emerg Infect Dis 17: 983-989.

45. Dale JB, Penfound TA, Chiang EY, Walton WJ (2011) New 30-valent M protein-based vaccine evokes cross-opsonic antibodies against non-vaccine serotypes of group A streptococci. Vaccine 29: 8175-8178. 